\title{
Biliary tract cancer in the chemical industry: a proportional mortality study.
}

\author{
P W BRANDT-RAUF ${ }^{1}$ J A HATHAWAY ${ }^{2}$ \\ From the Department of Medicine and Division of Environmental Sciences, ${ }^{1}$ Columbia-Presbyterian Medical \\ Center, New York, NY, and the Department of Medical Services, ${ }^{2}$ Chemicals Sector, Allied Corporation, \\ Morristown, NJ, USA
}

Carcinomas of the biliary tract are an uncommon but not rare form of cancer. They represent somewhat less than $1 \%$ of all malignant tumours and are the fifth most common cancer of the digestive tract. ${ }^{1}$ About 4500 deaths from cancers of the biliary tract are reported each year in the United States. ${ }^{2}$ Despite advances in diagnosis and treatment in recent years, biliary tract cancer is still associated with a dismal prognosis. Therefore, the identification of preventable causes has achieved some prominence.

One association that has been tentatively identified is between biliary tract cancer and work in certain industries. For example, an epidemiological survey of rubber workers in 1970 suggested an excess of cancer arising in the gall bladder and bile ducts. ${ }^{3}$ In a study of 1808 cases of biliary tract cancer from the California Tumor Registry gall bladder cancer was associated with the automotive, rubber, textile, and metal fabricating industries, and extrahepatic bile duct cancer (including carcinoma of the ampulla of Vater) was associated with the aircraft, automotive, rubber, wood finishing, and chemical industries. ${ }^{4}$ One of us has recently reviewed the necropsy experience with biliary tract cancer at Columbia-Presbyterian Medical Center in New York City for the past 40 years, and the findings were suggestive of such an association. ${ }^{156}$ Animal studies have shown an association between premalignant and malignant lesions of the biliary tract and exposure to various chemicals, including M-toluene diamine, aramite, 8-aminoazotoluene, 2-acetamidofluorene, and a polymer of 1,2-dihydro-2,2,4-trimethyl quinidine. ${ }^{7}$ We have, therefore, now reviewed the mortality data for Allied Corporation, currently a diversified company but historically a large chemical company, for any increase in biliary tract malignancies - in particular, extrahepatic bile duct cancer.

Accepted 21 January 1986

\section{Materials and methods}

The source of information was the mortality data file for Allied Corporation based in New Jersey. The file included the deaths of all active and retired employees from 1961 to the present based on insurance company records and over $90 \%$ of the records relate historically to the Allied Chemical Corporation. The file included sex, race, age at death, cause of death and associated findings, division and location in the company and job classification, year and place of death, date of hire, date last worked, and necropsy records. Since biliary tract cancer was not segregated out by site specific categories until the 8th International Classification of Diseases, analysis was restricted to deaths between 1968 and 1984. Furthermore, since the vast majority of records in the file were of white men $(83 \%)$, attention was focused on this group. Among the deaths on file, it is estimated that $84 \%$ relate to chemical plant employees and, among these, it is estimated that $85 \%$ would have had regular potential exposure to chemicals. Therefore, the mortality data file contains a total of 9253 recorded deaths of whom 5671 entries are for white men from 1968 to 1984 of whom 4049 or $71 \%$ would have had the potential for regular exposure to chemicals during their work experience. The potential chemical exposures are of a diverse nature; however, the chemical plants with the largest numbers of retirees and with the most death certificates on file were concerned with the production of soda ash, sulphuric acid, coke, and aromatic amine dyes.

The cases of death from biliary tract cancer were located and segregated by site-gall bladder, intrahepatic bile duct, extrahepatic bile duct, and ampulla of Vater. A proportionate mortality (PMR) analysis was performed on the cases among white men between 1968 and 1984 by dividing the number of deaths observed in each category by the number expected based on the proportion of deaths in this category in the United States general population ${ }^{8}$ and multiplying by 100 . The PMRs were adjusted for the 
potentially confounding effects of age, sex, race, and calendar time. The statistical significance of the PMRs was tested using Fisher's exact test and statistical significance was assumed for $p$ values less than 0.05 .

\section{Results}

Results are presented in the table. Of the recorded deaths among white male employees from 1968 to 198410 were from biliary tract cancer-four in the gall bladder, three in the ampulla of Vater, two in the intrahepatic bile ducts, and one in the extrahepatic bile ducts. Necropsy confirmation of the causes of death was available in three $(30 \%)$ of these cases. The average age at death was: gall bladder, 74 ; ampulla, 65; intrahepatic ducts, 70; extrahepatic ducts, 75 ; overall, 70 . Seven $(70 \%)$ of these cases had the potential of regular chemical exposure during their work experience. Most of these chemical exposures would have been to inorganic chemicals; only two of the cases worked at locations with possible organic chemical exposure (nylon manufacturing; research facility). No evidence of clustering by work site, job description, or likely exposure could be discerned.

The proportional mortality ratios for the gall bladder and the total biliary tract cancers approximated 100. The ratio for the extrahepatic bile duct cancers was considerably less than 100 , and the ratios for cancers of the intrahepatic ducts and the ampulla of Vater was considerably greater than 100 . None of these differences, however, achieved statistical significance.

In the complete mortality file were 17 cases of biliary tract cancer: seven in the gall bladder, four in the ampulla of Vater, two in the intrahepatic bile ducts, and four in extrahepatic bile ducts. Exact PMR analysis could not be performed on the whole group because sufficient data in the comparison population is not available. An analysis was performed by extrapolating existing data to the whole group, and the statistical significance of the observations remained unchanged. Once again, in the whole group $70 \%$ had potential for regular chemical exposure, mostly to inorganics, and no clustering by likely exposure was detected.

\section{Biliary tract cancer mortality by site}

\begin{tabular}{lcc}
\hline Site & No & $P M R$ \\
\hline Gall bladder & 4 & 101 \\
Ampulla of Vater & 3 & 280 \\
Intrahepatic ducts & 2 & 209 \\
Extrahepatic ducts & 1 & 27 \\
Total & 10 & 101 \\
\hline
\end{tabular}

PMR = Proportionate mortality.

\section{Discussion}

The death certificate study previously cited noted an increase of extrahepatic bile duct cancer including ampullary cancer in the chemical industry. ${ }^{4} \mathrm{~A}$ previously reported necropsy series indicated that occupational or environmental exposure might be important in biliary tract cancer. ${ }^{156}$ The findings here indicate that in one major chemical company at least no form of biliary tract cancer is statistically significantly increased. In this company there may not have been exposure to the as yet unidentified chemicals responsible or the exposure may have been minimised, either due to numbers exposed or degree of exposure, to the point of being insignificant in terms of health effects. It is recognised that in this instance only one chemical company is represented and small numbers of cases are concerned; the power of this study to detect a twofold increase at a $95 \%$ confidence level was only $20 \%$ due to the small numbers of deaths from biliary tract cancer in this population. Nevertheless, for two of the four sites the PMR was considerably greater than 100 , although for the case of extrahepatic bile duct cancer it was considerably less than 100; furthermore, a similar pattern of mortality has been seen with other industry mortality data. ${ }^{6}$ These disparate findings serve to underscore the need for further research in this area. It is especially important to pursue the identification of the particular chemicals that might be concerned or the nature of any confounding variables that could explain the differences in the studies noted in the hope of preventing further cases. This is particularly important in light of the ineffectual treatment and dismal prognosis associated with these malignancies.

\section{References}

${ }^{1}$ Brandt-Rauf PW, Pincus MR, Adelson S. Cancer of the gallbladder: review of 43 cases. Human Pathol 1982;13:48-53.

${ }^{2}$ Fraumeni JF. Cancers of the pancreas and biliary tract: epidemiologic considerations. Cancer Res 1975;35:3437-46.

${ }^{3}$ Mancuso TF, Brennan MJ. Epidemiologic considerations of cancer of the gallbladder, bile ducts and salivary glands in the rubber industry. J Occup Med 1970;12:333-41.

${ }^{4} \mathrm{~K}$ rain LS. Gallbladder and extrahepatic bile duct carcinoma. Geriatrics 1972;27:111-7.

${ }^{5}$ Brandt-Rauf PW, Pincus MR, Adelson S. Carcinoma of the extrahepatic bile ducts: some new perspectives. Survey of Digestive Diseases 1984;2:156-63.

${ }^{6}$ Brandt-Rauf PW, Pincus MR. Biliary tract cancer: some new perspectives. In: Abstracts of the spring national meeting of the American Society of Clinical Pathologists and College of American Pathologists, Chicago, Illinois, March 30-April 14, 1985. Chicago, ASCP/CAP, 1985:26.

${ }^{7}$ Greenwald ED, Greenwald ES, Brenner SM. Extrahepatic bile duct cancer. NY State J Med 1981;81:324-6.

${ }^{8}$ Division of Vital Statistics Statistical Resources Branch. Deaths from each cause by 5-year age groups, race, and sex: United States. Hyattsville, Md, National Center for Health Statistics, 1984. 